\title{
Research of LED lamp constant current driver software system
}

\author{
Liu ling \\ XI'AN University of Arts and Science \\ xi'an, China, 710065 \\ liuxuanping2003@yahoo.com.cn
}

\begin{abstract}
The LED lamps replace traditional lamps is the direction of development domestic and abroad.It mainly introduced the LED lamp brightness control method from the aspect of software, then research and production of the LED efficient drive based on a IC chip digital boost constant current regulator, for the actual driving high power LED. It was realized 6 level control on the brightness of LED. The microcontroller controls D/A converter to make the output different voltage values through software programming, and then adjusts the DC/DC conversion circuit current size, finally implements LED brightness control. The experimental makes it possible to have the lamp with adjustable brightness, energy saving, achieve a wide range of voltage, current constant and so on.
\end{abstract}

Keywords- LED lamp;Constant current drive;Single chip microcomputer; digital boost constant current regulator.

\section{INTRODUCTION}

For global lighting industry, 2012 is an unusual year because the government around the world starts to promote energy-saving policies and ban incandescent lamps. Meanwhile, the cost of LED lights keeps falling. All these advantages help to stimulate the development of the energysaving market. As the energy shortage raises energy-saving awareness around the world, incandescent lamps have to fade out from lighting industry. EU, USA, Japan, China have worked out the roadmap of banning incandescent lamps. Europe's households have set off widely banning inefficient 60 watt light bulbs from September 1st 2011.The inefficient 60 watt light bulbs are taken place of CFLs and LEDs. And European Union legislation will ban the manufacture and import of all incandescent bulbs since September 1st 2012. America starts to phase out traditional 100 watt incandescent light on January 1st. Japanese government has encouraged major electronics retailers and home appliance makers to voluntarily halt production and sales of incandescent bulbs to save power this summer. And Panasonichas announced to stop production of incandescent bulbs used in household market before October 31th, 2012.The Chinese government announced a time frame for the phasing-out of incandescent lamps.

China will ban imports and sales of $100 \mathrm{~W}$ and higher incandescent bulbs starting from October 1st, 2012, and ban on $60 \mathrm{~W}$ and higher incandescent bulbs starting October 1st from October 1st, 2015 to September 30th,2014. The time will serve as an evaluation period in 2016. At present a lot of enterprises are optimistic about the LED of this sunrise industry development space ,also accelerate a LED technology's breakthrough in our country, the LED lights gradually replaced the white matter light is an inevitable trend in the future.

\section{BLOCK DIAGRAM OF SYSTEM HARDWARE}

System overall plan design include:the design of DC/DC driving circuit, sampling, a voltage reference and feedback circuit design, single-chip control circuit design. Scheme diagram as shown in figure 1.

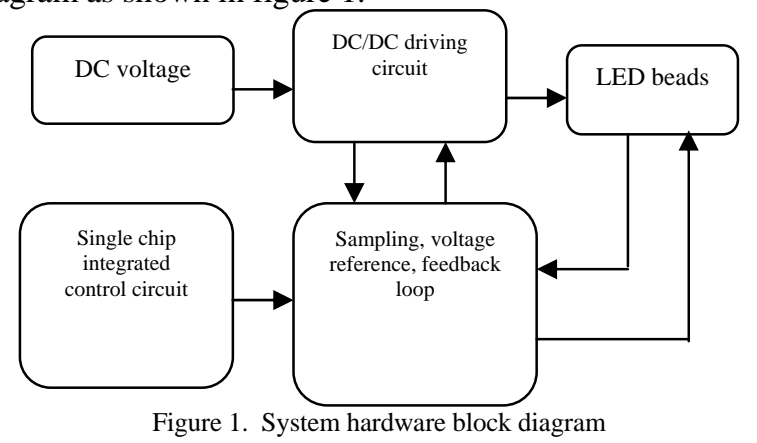

\section{SySTEM HARDWARE CIRCUIT DESIGN}

\section{A. Chip profile}

The AT89S52 is a low-power, high-performance CMOS 8-bit microcontroller with $8 \mathrm{~K}$ bytes of in-system programmable Flash memory. The device is manufactured using Atmel's high-density nonvolatile memory technology and is compatible with the industry-standard 80C51 instruction set and pinout. The on-chip Flash allows the program memory to be reprogrammed in-system or by a conventional nonvolatile memory programmer.

The Idle Mode stops the CPU while allowing the RAM, timer/counters, serial port, and interrupt system to continue functioning. The Power-down mode saves the RAM contents but freezes the oscillator, disabling all other chip functions until the next interrupt or hardware reset.

\section{B. Hardware circuit}

As shown in Figure 2,a single chip AT89S52 reset circuit is made of the K1 key, C8 capacitor and R14, R16 resistor. MCU reset circuit consists of a power on reset, button level reset and button reset pulse.

The system selects the button level reset, it is realize that supply is switched on through the reset terminal of the resistor RST R16 and Vcc power. During the button level reset,the reset signal ensure high level duration longer than 2 machine cycles. 
The AT89S52 chip's clock circuit composed of an oscillating circuit and a frequency dividing circuit.The oscillating circuit are made of an inverter, external parallel quartz crystal and a capacitor for generating an oscillating pulse. The divider circuit uses oscillating pulse frequency resolution, to obtain the requiring clock signal. Its high gain inverting amplifier has the input end pin XTAL1 and the output pin XTAL2. It were connected with the quartz crystal oscillator(oscillation frequency of $12 \mathrm{MHz}$ )and a two capacitor(capacitor C11 and C12 from 22pF)on the chip through these two pins. The quartz crystal as a sensitive element and capacitor constitute oscillation loop for which on-chip amplifier provide with positive feedback and oscillation of the desired phase shift conditions, thereby form a stable self-excited oscillator.

It is not used directly for the microcontroller but to be divided that the oscillation signal is generated by the oscillation circuit, the frequency can be various microcontroller associated clock signal, as shown in figure 2.Oscillation pulse is as the system clock signal by two divider, it generates ALE signal divided by three on the basis of the two divider, and get the machine cycle signal divided by six on the basis of two divider.The P0 port is used as the data sent to the D/A converter, the P0 port hasn't pull-up supreme resistor internal, therefore connection with an external pull-up resistor RST1.

\section{D/A conversion circuit}

The D/A conversion circuit's chip is DAC0832,it is fully compatible with microprocessors.D/A converter are made of the 8 bite input latch,8-bit DAC register, 8 D/A conversion circuit and conversion control circuit.

The system adopt to DAC0832 straight.They are grounded low-level access GND that the chip select signal CS, the data latch strobe input pin WR1,the data transfer control signal input pin XFER and DAC register strobe input pin WR2.The data latched allow eighth pin is reference voltage of Vref, the reference voltage is $2.5 \mathrm{~V}$ if you adjust external sliding rheostat R15.Because of the chip DAC0832 being a form of current output, you have to add an op-amp that it convert the current to voltage directly.

The converted voltage is a negative pressure in the external,then through the op-amp OP07 constituted reverse proportional amplifier, where is the OP07 magnification such as the equation (1) below:

$$
A=-\frac{R_{12}}{R_{13}}
$$

By equation (1) and a circuit diagram in Figure 2 shows that the magnification is $A=-\frac{R_{12}}{R_{13}}=-3.3$.Therefore, it is converted to the circuit by the chip output of negative voltage, to achieve the desired $\mathrm{D} / \mathrm{A}$ input voltage required.It is provided the $\mathrm{D} / \mathrm{A}$ input voltage range of $0 \mathrm{~V} \sim 4.7 \mathrm{~V}$ by the DC / DC converter circuit of the current regulation circuit.

\section{Negative pressure generating circuit}

The circuit turns a positive input voltage into the negative output voltage adopting the DC / DC converter and the chip ICL7660S because the amplifier OP07 works in the negative voltage state in the D/A conversion circuit,where is the opposite polarity of Vin and Vout. The circuit achieves the voltage polarity converter using the oscillator and multichannel analog switches, thus quiescent current small, high conversion efficiency, simple peripheral circuit.

As shown in Figure 2.The desired negative voltage is made of an ICL7660S plus the three 10uF capacitor. The capacitor $\mathrm{C} 9, \mathrm{C} 10, \mathrm{C} 12$ is the $10 \mathrm{uF}$ tantalum capacitor that has leakage small and low dielectric loss, in order to improve the efficiency of power conversion. When is Vcc $<+6.5 \mathrm{~V}, 5$ feet can be directly used as output; When is Vcc $>+6.5 \mathrm{~V}$, the output circuit is connected in series to a fast diode to avoid damage chip.

\section{The System SofTWARE Programming FLOW CHART}

The program adjust to the LED lamp beads brightness through the control of the key K2 and K3. When you do press the K3 key each time, $X$ increased by 20,where is $X$ LED brightness variable, then the LED brightness is enhanced; that the control signal input pin is connected to the high level Vcc, such DAC0832 work being the straight way. When you do press the K2, X reduced by 20, thus the LED

brightness is weakened.

The system reads the appropriate values which it compared with the procedures set value, whether it is determined the press K2 or K3.The PWM signal is changed by the microcontroller intermittent so as to change the load current value (a small value of the current of the electromagnet), while the microcomputer does other work in most of the time, the load current value can be maintained unchanged during this time until the next variable flow operation.

The set current value is multiplied by 20 whether the output current meet the requirements or not, so as to achieve the purpose of the constant current. If the output voltage and current is not normal a PWM signal with $0 \%$ duty cycle would deliver by the microcontroller immediately ,the power supply stop the output, and prevent damage from the power supply and LED lighting.If the microcontroller receives fault detection instruction a central control unit returns a fault response at this time for the power supply and lighting maintenance conveniently.

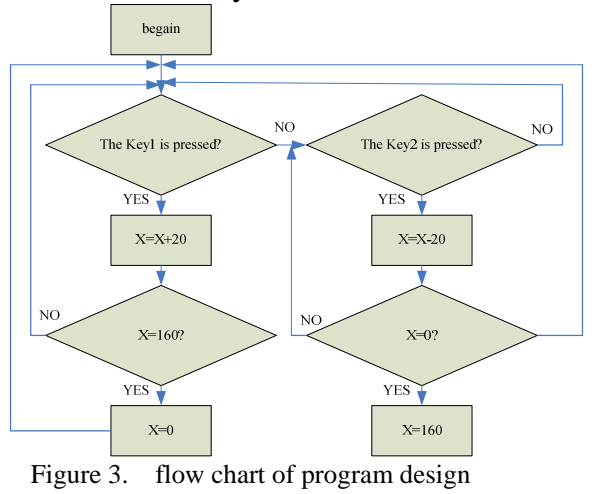

If the system receives a dimming command that it will enter the interrupt subroutine and realize dimming function 
in a sampling period.The microcomputer control PWM regulator current through the open loop,there is no strict requirements of the load current value and the expected microcomputer signal output,there is the positive correlation between the upper limit (the actual current is greater than a certain value ) and lower ( full power failure) restrictions.The system control the DC/DC circuit corresponding output current by analog dimming circuit.

\section{EXPERIMENTAL RESULTS}

\section{A. DC-DC each main index test results}

Table 1 is DC-DC each main index test results.

B. The brightness of the lamp and $4.5 \mathrm{~V}$ battery use time

The $4.5 \mathrm{v}$ battery continuously supply for 86 hours. The similar products can only consecutively work for maximum 10 hours on the market, for there are always a significant reduction in electricity losses.

Table 1 the main index of DC-DC

\begin{tabular}{|c|l|c|}
\hline number & \multicolumn{1}{|c|}{ Test item } & Test results \\
\hline 1 & Output voltage range & $0 \mathrm{~V} \sim 12 \mathrm{~V}$ \\
\hline 2 & $\begin{array}{l}\text { Maximum output } \\
\text { current }\end{array}$ & $197 \mathrm{~mA}$ \\
\hline 3 & Load regulation & $0.1 \%$ \\
\hline 4 & Output noise ripple & $\mathrm{Vpp}=10 \mathrm{mV}$ \\
\hline 5 & $\begin{array}{l}\text { DC-DC conversion } \\
\text { efficiency }\end{array}$ & $97.1 \%$ \\
\hline
\end{tabular}

\section{CONCLUSION}

The project achieve the multiple LED current value of the control, there can mix the four kinds of brightness with a dimmer. Based on the above conclusions,the system can be applied to many occasions at multiple LED control, such as surgical lights, home lighting, large outdoor lighting design, as well as hand-held flashlight. It can achieve the purpose of energy saving,then have low energy consumption, and high efficiency, The LED lamp industry has a certain great meaning.

\section{ACKNOWLEDGMENT}

Thanks to the strong support of the Xi'an Science and Technology Project No. CX12189WL12!

\section{REFERENCES}

[1] Jiann-Jong Chen.Bo-Han Hwang.Yao-Ren Guo.Yuh-Shyan Hwang.Cheng-Chieh Yu. A high-efficient WLED driver using lightbalanced-controlled techniques with current-locked loops. Analog Integr Circ Sig Process (2012) 72:363-373

[2] Ran Chen, Dongye Sun and Wen-Pei Sung,Frontiers of Advanced Materials and Engineering Technology ,Advanced Materials Research January, 2012:430-432

[3] XU Gen-da, ZHAO He-chang. Design of high efficiency power supply for high-power LED. Chinese Journal of Power Sources.July 2011:811 813;

[4] SONG Xiang-rong, SHU Jie, WU Qi-bao. LED power drive based on a double-ring switch control. Chinese Journal of Power Sources.November 2011:1418 1420;

[5] YIN Wei, XING Li-dong, QIAN Zhi-yu.The Design and Implementation of Multi-channel lighting LED Dimming Control Circuit. Industrial Applications and Communications.August 2011:94 97

[6] ZHAN Jun, MA Xiao- jun. Research and Design of White LED Driver Based on DC/DC. Advanced Display.May 2008:54 56

[7] Massimiliano Belloni.Edoardo Bonizzoni.Piero Malcovati.Franco Maloberti.A high efficiency 4-output single inductor DC-DC buck converter with self boosted snubber. Analog Integr Circ Sig Process (2011) 67:169-177

[8] Ismail Atacak.Omer Faruk Bay. A type-2 fuzzy logic controller design for buck and boost DC-DC converters. J Intell- Manuf (2012) 23:1023-1034

[9] Hongxing Li.Tsutomu Wakimoto .Keita Murase.Kenji Tomiyoshi.0.25LM1.2 MHz BOOST-PFM-CONTINUOUS triple mode LED driver integrated in analog front end IC for portable application. Analog Integr Circ Sig Process (2012) 72:595-603

[10] Viktor Avrutin .Enric Fossas.Albert Granados.Michael Schanz.Virtual orbits and two-parameter bifurcation analysis in a ZAD-controlled buck converter. Nonlinear Dyn (2011) 63: 19-33

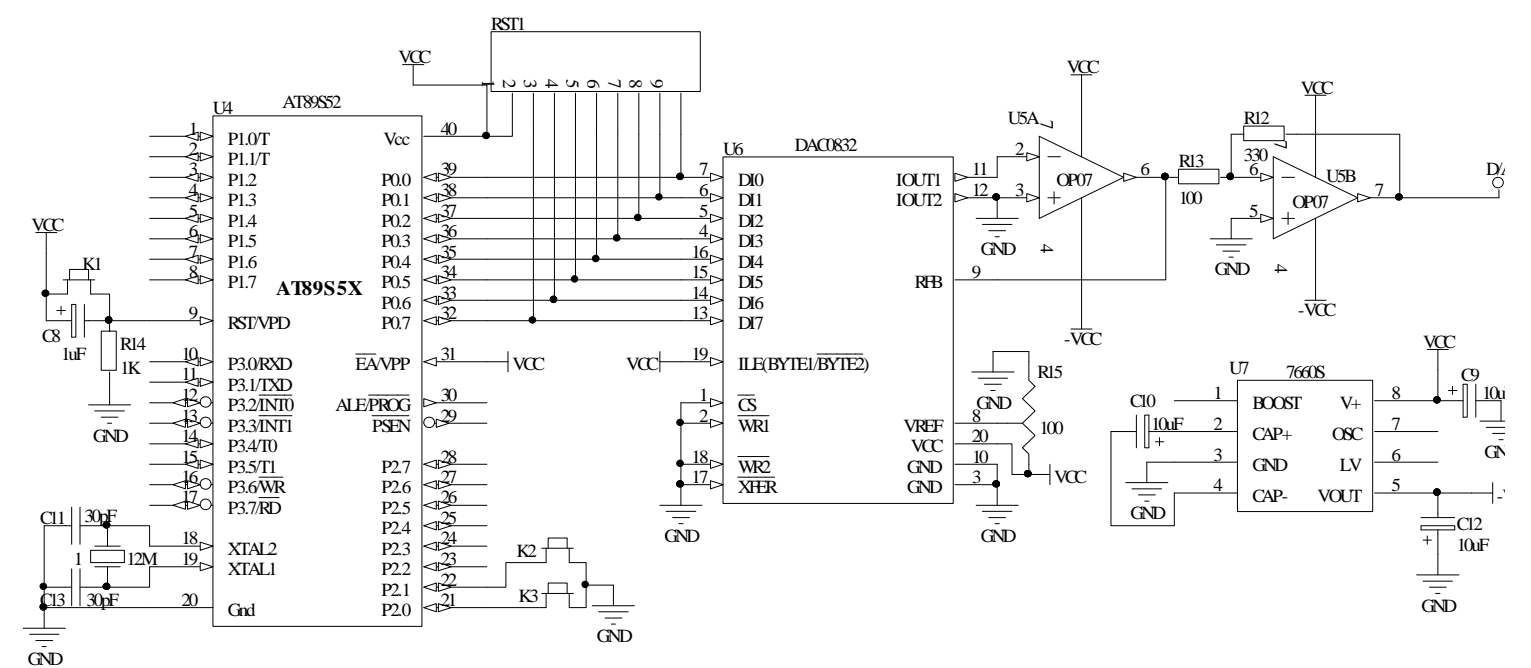

Figure 2. AT89S52 microcontroller peripheral circuit and control circuit 